\title{
NORMSETS OF ALMOST DEDEKIND DOMAINS AND ATOMICITY
}

\author{
RICHARD ERWIN HASENAUER
}

\begin{abstract}
In this paper, we will introduce a new norm map on almost Dedekind domains. We compare and contrast our new norm map to the traditional Dedekind-Hasse norm. We prove that factoring in an almost Dedekind domain is in one-to-one correspondence to factoring in the new normset, improving upon this results in [1]. In [4], an atomic almost Dedekind domain was constructed with a trivial Jacobson radical. We pursue atomicity in almost Dedekind domains with nonzero Jacobson radicals, showing the usefulness of the new norm we introduced. We state theorems with regard to specific classes of almost Dedekind domains. We provide a necessary condition for an almost Dedekind domain with nonzero Jacobson radical to be atomic.
\end{abstract}

1. Introduction. The study of factorization in Dedekind domains has a rich history, going all the way back to Gauss. We say $b$ is irreducible (an atom) if $b=c d$ implies $c$ or $d$ is a unit. We say $D$ is atomic if every nonzero element factors into a finite product of atoms. Recall that, if $D$ is a Dedekind domain, then $D$ is atomic. Further, any nonzero ideal of a Dedekind domain can be factored uniquely as a product of positive powers of maximal ideals. Two concepts developed to study factorization in Dedekind domains are the Dedekind-Hasse norm and the ideal class group.

Let $D$ be a domain with maximal ideals $\operatorname{Max}(D)$. Recall $D$ is Dedekind if $D$ is Noetherian and every localization $D_{M}$ is a Noetherian valuation domain. If we drop the assumption that $D$ is Noetherian we get a domain that is called almost Dedekind. More precisely, $D$ is almost Dedekind if $D_{M}$ is a Noetherian valuation domain for all $M$ in $\operatorname{Max}(D)$. Thus, an almost Dedekind domain $D$ is Dedekind if and only if it is Noetherian.

2010 AMS Mathematics subject classification. Primary 13A50, Secondary $13 \mathrm{~F} 15$.

Keywords and phrases. Factorization, almost Dedekind.

Received by the editors on October 3, 2013, and in revised form on September 5, 2014.

DOI: $10.1216 / \mathrm{JCA}-2016-8-1-61$

Copyright (C)2016 Rocky Mountain Mathematics Consortium 
In a Dedekind domain, every ideal can be written uniquely as a finite product of maximal ideals. In [7], the authors sought to generalize this ideal factorization property to almost Dedekind domains. Dedekind domains come equipped with a nice norm, the Dedekind-Hasse norm.

One of the questions we pursued was to what extent can we construct a norm on an almost Dedekind domain. We relaxed a few of the traditional requirements for a norm and were able to construct an object that yields results with respect to atomicity. Since all Dedekind domains are atomic, it seems natural to ask which almost Dedekind domains are atomic?

Let $F$ denote a finite extension of $\mathbb{Q}$, the rational numbers, with a ring of integers $R$. Let $S$ be the image of the Dedekind-Hasse norm of $R$. Coykendall, in [1], showed if $F$ is Galois over $\mathbb{Q}$, then $R$ is a unique factorization domain (UFD) if and only if $S$ is a unique factorization monoid (UFM). We use our new norm to obtain a similar result for almost Dedekind domains. In fact, if we restrict our norm to Dedekind domains, our result improves upon Coykendall's result, albeit with a different norm.

In [4], an atomic almost Dedekind domain was constructed. This particular domain has a trivial Jacobson radical. We ask whether we can construct an atomic almost Dedekind domain with a nonzero Jacobson radical. Dedekind domains with nonzero Jacobson radicals are merely semi-local PIDs. With respect to this thinking, one might think that constructing an almost Dedekind domain with a nonzero Jacobson radical that is atomic might not be too heavy a task. However, the opposite seems to be true. We walk the reader through a series of proofs, arriving at what seems to be an unusual necessary condition for such a domain to be atomic.

2. A new norm. One of our goals was to construct an object that would help us explore which almost Dedekind domains are atomic. The only real thing at our disposal is the fact that, if $D$ is almost Dedekind, then $D_{M}$ is a Noetherian valuation domain for all $M \in \operatorname{Max}(D)$. In essence, what we do is take these local valuations and piece them together to form a global object we call the norm. We wish to do this in a way that will allow us to take advantage of the additive and multiplicative properties. That is, if $\nu$ is a valuation, then 
$\nu(a b)=\nu(a)+\nu(b)$ and $\nu(a+b) \geq \min \{\nu(a), \nu(b)\}$ where the inequality is strict if $\nu(a) \neq \nu(b)$ and $a+b \neq 0$.

Making these notions more formal we let $D$ be an almost Dedekind domain with maximal ideals $\operatorname{Max}(D)$. Now, for every $M \in \operatorname{Max}(D)$, we have a map

$$
\nu_{M}: D_{M} \longrightarrow \mathbb{N}_{0}
$$

It is well known that

$$
D=\bigcap_{M \in \operatorname{Max}(D)} D_{M}
$$

Now, for $b \in D$, we have $\nu_{M}(b)=0$ if $b \notin M$ and $\nu_{M}(b)>0$ if $b \in M$.

Definition 2.1. For nonzero $b \in D$, we define the norm of $b$ to be the net

$$
N(b)=\left(\nu_{M}(b)\right)_{M \in \operatorname{Max}(D)} \subseteq \prod_{M \in \operatorname{Max}(D)} \mathbb{N}_{0}
$$

If $u$ is a unit in $D$, then $N(u)$ is the zero net. As mentioned earlier, our new norm does not satisfy the conditions of a traditional norm, but we refer to our map as a norm for convenience and its norm-like properties. In fact, we will see that in many ways our norm is superior to the traditional Dedekind-Hasse norm.

We can define our local valuations on $K$ the field of fractions of $D$. In this case, $\nu_{M}: K \rightarrow \mathbb{Z}$. Under this definition, we see that $k \in K$ is in $D$ if and only if, for all $M, \nu_{M}(k) \geq 0$. We will need this notion for one of our theorems.

Now, in [1], the normset associated with the traditional DedekindHasse norm was explored. The normset with the Dedekind-Hasse norm forms a monoid. We wish to parallel this result so we need to define the addition between $N(a)$ and $N(b)$. We define the addition of nets componentwise. That is,

$$
N(a)+N(b):=\left(\nu_{M}(a)+\nu_{M}(b)\right)_{M \in \operatorname{Max}(D)} .
$$

Under this definition and the assumption that $D$ is unital, we will see the image forms a monoid. 
Definition 2.2. Let $D$ be an almost Dedekind domain. We define the normset of $D$ to be

$$
\operatorname{Norm}(D)=\{N(b) \mid b \in D, b \neq 0\} .
$$

As mentioned before, we want to take full advantage of the properties of valuations. We get the following theorem solely from the properties of valuations.

Theorem 2.3. Let $D$ be an almost Dedekind domain. For all $a, b \in D$ we have $N(a b)=N(a)+N(b)$.

$$
\text { Proof. } N(a b)=\left(\nu_{M}(a b)\right)_{M \in \operatorname{Max}(D)}=\left(\nu_{M}(a)+\nu_{M}(b)\right)_{M \in \operatorname{Max}(D)}=
$$
$N(a)+N(b)$.

Now the image of the Dedekind-Hasse norm over $\mathbb{Q}$ is contained in $\mathbb{Z}$; thus, it is easy to see which elements are "smaller" than others. We will want something similar with our norm so, we define a partial ordering on Norm $(D)$. Just as with the order in the Dedekind-Hasse normset, the use of this partial ordering will yield results with respect to factorization.

Definition 2.4. We say $N(a) \leq N(b)$ if, for all $M \in \operatorname{Max}(D)$, we have $\nu_{M}(a) \leq \nu_{M}(b)$. We say $N(a)<N(b)$ if $N(a) \leq N(b)$, and there exists an $M \in \operatorname{Max}(D)$ with $\nu_{M}(a)<\nu_{M}(b)$.

It should be noted that two elements are not necessarily comparable within this order, that is, there may be $a, b \in D$ such that $N(a) \not \leq N(b)$ and $N(b) \not \leq N(a)$, this will be of little limitation. We present a very powerful lemma; the result's veracity is due to the local behavior of almost Dedekind domains.

Lemma 2.5. Let $D$ be an almost Dedekind domain, and let $a, b \in D$. $N(a) \leq N(b)$ if and only if a divides $b$.

Proof. Suppose $N(a) \leq N(b)$. We have $b / a$ is in the quotient field of $D$. Now $\nu_{M}(b / a)=\nu_{M}(b)-\nu_{M}(a) \geq 0$ for all $M \in \operatorname{Max}(D)$. Thus, $b / a \in D_{M}$ for all $M$. Hence, $b / a \in D$. We conclude that $a$ divides $b$. Suppose $a$ divides $b$. Then $b / a \in D$. Thus, $b / a \in D_{M}$ for 
all $M \in \operatorname{Max}(D)$. Hence, for all $M$, we have $\nu_{M}(b / a) \geq 0$. Thus, $\nu_{M}(b) \geq \nu_{M}(a)$, and we conclude that $N(a) \leq N(b)$.

This result is not true for the traditional Dedekind-Hasse norm. Let $N^{\prime}$ denote the Dedekind-Hasse norm. Recall if $a \mid b$ in a Dedekind domain, then $N^{\prime}(a) \mid N^{\prime}(b)$. However, it is not true that $N^{\prime}(a) \mid N^{\prime}(b)$ implies $a \mid b$. To see this, let us consider an example.

Example 2.6. Consider $D=\mathbb{Z}[\sqrt{-14}]$. The traditional norm is $N^{\prime}(a+b \sqrt{-14})=a^{2}+14 b^{2}$. Now $N^{\prime}(5+2 \sqrt{-14})=81$ and $N^{\prime}(3)=9$ but 3 does not divide $5+2 \sqrt{-14}$. But what about our norm? Let

$$
M_{1}=(3,5+2 \sqrt{-14})
$$

and

$$
M_{2}=(3,5-2 \sqrt{-14}) .
$$

It is easy to see that $M_{1}$ and $M_{2}$ are maximal in $D$ and $M_{1} M_{2}=(3)$. That is, we have $\nu_{M_{1}}(3)=1$ and $\nu_{M_{2}}(3)=1$. Now $5+2 \sqrt{-14} \in$ $M_{1}$, but $5+2 \sqrt{-14} \notin M_{2}$. If $5+2 \sqrt{-14} \in M_{2}$, we would have $5+2 \sqrt{-14}+5-2 \sqrt{-14}=10 \in M_{2}$ but 3 and 10 are coprime in $D$. Therefore, we would have $1 \in M_{2}$. Thus, $5+2 \sqrt{-14} \notin M_{2}$. In addition, we have $\nu_{M_{2}}(5+2 \sqrt{-14})=0$. We conclude that $N(3) \not \leq N(5+2 \sqrt{-14})$. Thus, our norm recognizes that 3 is not a divisor of $5+2 \sqrt{-14}$.

We now make some structural observations about Norm $(D)$. We observe that the normset is a monoid, which is in direct parallel to the Dedekind-Hasse normset.

Theorem 2.7. Let $D$ be an almost Dedekind domain. Then $\operatorname{Norm}(D)$ forms an additive commutative monoid whose elements are cancellative.

Proof. First the zero net is in Norm $(D)$ because $D$ has an identity. Now, if $N(a)$ and $N(b)$ are in $\operatorname{Norm}(D)$ (that is, $a, b \in D$ ), then $N(a)+N(b)=N(a b) \in \operatorname{Norm}(D)$ because $a b \in D$. It is easy to prove that $\operatorname{Norm}(D)$ is commutative and that every element of Norm $(D)$ is cancellative. 
The Dedekind-Hasse norm gives great insight into which integers appear in the normset. For example, in the case of the Gaussian integers $\mathbb{Z}[i]$, the Dedekind-Hasse norm is $N^{\prime}(a+b i)=a^{2}+b^{2}$. Thus, the normset consists of all integers that can be expressed as the sum of two squares. We wish to classify the element of our new normset in a similar fashion.

Theorem 2.8. Let $D$ be an almost Dedekind domain with $\operatorname{Max}(D)=$ $\left\{M_{\lambda}\right\}_{\lambda \in \Lambda}$. Then

$$
\left(e_{\lambda}\right)_{\lambda \in \Lambda} \in \operatorname{Norm}(D) \Longleftrightarrow \bigcap_{\lambda \in \Lambda} M_{\lambda}^{e_{\lambda}} \text { is a principal ideal, }
$$

where we take $M^{0}=D$.

Proof. For the forward direction we take $a \in D$ with $N(a)=$ $\left(e_{\lambda}\right)_{\lambda \in \Lambda}$. Recall $\nu_{M_{\lambda}}(a)=e_{\lambda}$ is equivalent to saying $a \in M_{\lambda}^{e_{\lambda}}$ and $a \notin M_{\lambda}^{e_{\lambda}+1}$. Thus, for all $\lambda$ we have $a \in M_{\lambda}^{e_{\lambda}}$; hence, $a \in \cap_{\lambda \in \Lambda} M_{\lambda}^{e_{\lambda}}$. We will show this ideal is actually $(a)$. Suppose $b \in \cap_{\lambda \in \Lambda} M_{\lambda}^{e_{\lambda}}$. Then, for all $\lambda$, we have $\nu_{M_{\lambda}}(b) \geq e_{\lambda}=\nu_{M_{\lambda}}(a)$. In other words, we have $N(a) \leq N(b)$; hence, $a$ divides $b$. We conclude that

$$
(a)=\bigcap_{\lambda \in \Lambda} M_{\lambda}^{e_{\lambda}} .
$$

For the other direction, we suppose $(a)=\cap_{\lambda \in \Lambda} M_{\lambda}^{e_{\lambda}}$. Then $N(a)=$ $\left(e_{\lambda}\right)_{\lambda \in \Lambda}$.

Since the image under our norm map is well behaved in a structural sense, one might ask the question, if we impose more conditions on $D$ what is the effect on $\operatorname{Norm}(D)$ ?

Now we will show that $X$ is a factorization property of $D$ if and only if $X$ is a factorization property of $\operatorname{Norm}(D)$.

We denote the (multiplicative) monoid of nonzero elements of $D$ by $D^{\bullet}$ and the set of units of $D$ by $D^{\times}$.

Theorem 2.9. Let $D$ be an almost Dedekind domain. Then $D^{\bullet} / D^{\times} \cong$ $\operatorname{Norm}(D)$. 
Proof. Let $\phi: D^{\bullet} / D^{\times} \rightarrow \operatorname{Norm}(D)$ be defined by $\phi\left(x D^{\times}\right)=N(x)$ for all $x \in D^{\bullet}$. We show that $\phi$ is a monoid isomorphism. Let $x, y \in D^{\bullet}$. Then $x D^{\times}=y D^{\times}$if and only if $x=u y$ for some $u \in D^{\times}$which is if and only if $N(x)=N(u y)=N(u)+N(y)=N(y)$ Therefore, $\phi$ is well defined and injective. It follows from Theorem 2.3 that $N$ is a monoid homomorphism. Clearly, $N$ is surjective. Consequently, $\phi$ is a monoid epimorphism.

Now let $X$ be a factorization property (i.e., atomic, UFD, HFD, etc.). Using the previous theorem we obtain that $D$ satisfies $X$ if and only if $D^{\bullet} / D^{\times}$satisfies $X$ if and only if $\operatorname{Norm}(D)$ satisfies $X$. This gives us the following theorem.

Theorem 2.10. Let $D$ be an almost Dedekind domain, and let $X$ be a factorization property. $D$ satisfies $X$ if and only if $\operatorname{Norm}(D)$ satisfies $X$.

3. Properties of atomic almost Dedekind domains. We wish to pursue what properties are necessary for an almost Dedekind domain to be atomic. The only atomic almost Dedekind domain that appears in the literature is one with a trivial Jacobson radical. See [4]. What role does the Jacobson radical play in atomicity? If a Dedekind domain has a nonzero Jacobson radical, then it is a semi-local PID. We will see that this nice property does not extend to the class of almost Dedekind domains.

We take definitions from [7]. We say a maximal ideal is sharp if it is the radical of a finitely generated ideal. Maximal ideals that are not sharp are called dull. An almost Dedekind domain that contains only dull maximal ideals will be referred to as a dull domain.

Definition 3.1. We say an almost Dedekind domain $D$ is bounded, or a bounded domain, if, for all nonzero $b \in D$, we have $b$ is of bounded norm. That is, there exists a $\rho \in \mathbb{N}$ such that $\nu_{M}(b)<\rho$ for all $M \in \operatorname{Max}(D)$. We will call atoms of bounded norm, bounded atoms.

Both glad and $S P$-domains are bounded (see $[\mathbf{5}, \mathbf{8}]$.) We will call domains that are not bounded, unbounded domains. Unbounded almost 
Dedekind domains can be constructed. We present an example that relies on the following theorem from [3].

Theorem 3.2. Let $D$ be a Dedekind domain with quotient field $K$, and let $\left\{P_{i}\right\}_{i=1}^{r},\left\{Q_{i}\right\}_{i=1}^{s}$ and $\left\{U_{i}\right\}_{i=1}^{t}$, where $r \geq 1$, be three collections of distinct maximal ideals of $D$, each with finite residue field. Then there exists a simple quadratic extension field $K(t)$ of $D$ with $t$ integral over $D$ and separable over $K$ such that if $\bar{D}$ is the integral closure of $D$ in $K(t)$, each $P_{i}$ is inertial with respect to $\bar{D}$, each $Q_{i}$ ramifies with respect to $\bar{D}$, and each $U_{i}$ decomposes with respect to $\bar{D}$.

Example 3.3 (The $D^{\infty}$ domain). Let $D=\mathbb{Z}_{(q)}$ for some odd prime $q$. Let $K$ denote the quotient field of $D$. We can split $(q)$ by adjoining $t_{1,1}$ a root of $x^{2}-p$ for some prime $p \neq q$ that is a square modulo $q$. Let $K_{1}=K\left[t_{1,1}\right]$. We have $(q)=\left(q_{1}\right)\left(q_{1,2}\right)$, where $\left(q_{1}\right)$ and $\left(q_{1,2}\right)$ are distinct. We set $D_{1}$ to be the integral closure of $D$ in $K_{1}$. For the remainder of the construction $D_{i}$ will be the integral closure of $D_{i-1}$ in $K_{i}$ and the $t_{i, j}$ are elements of the algebraic closure of $K$. Now, by Theorem 3.2 , we can find $t_{2,1}$ such that $\left(q_{1,2}\right)=\left(q_{2,1}\right)\left(q_{2,2}\right)$ in $D_{1}\left[t_{2,1}\right]$ while $\left(q_{1}\right)$ remains inert.

Now we find $t_{2,2}$ such that $\left(q_{2}\right)^{2}=\left(q_{2,1}\right)$ and the other two primes remain inert in $K_{2}=K_{1}\left[t_{2,1}, t_{2,2}\right]$. First, we set $D_{2}$ to be the integral closure of $D$ in $K_{2}$. Next, we split $\left(q_{2,2}\right)=\left(q_{3,1}\right)\left(q_{3,2}\right)$ via another simple quadratic extension (add $\left.t_{3,1}\right)$ while keeping the three other primes inert. We then ramify $\left(q_{3,1}\right)$ twice by using two simple quadratic extensions adding $t_{3,2}, t_{3,3}$ (we keep all other primes inert), thus yielding $\left(q_{3}\right)^{4}=\left(q_{3,1}\right)$.

Now we set $K_{3}=K_{2}\left[t_{3,1}, t_{3,2}, t_{3,3}\right]$. First, we set $D_{3}$ to be the integral closure of $D$ in $K_{3}$. We continue by induction. In $D_{i}$, we have $i+1$ maximal ideals, namely, $\left(q_{1}\right),\left(q_{2}\right), \ldots\left(q_{i}\right)$ and $\left(q_{i, 2}\right)$. Second, we add $t_{i, 1}$ that splits $\left(q_{i, 2}\right)=\left(q_{i+1,1}\right)\left(q_{i+1,2}\right)$. Third, we adjoin elements $t_{i, 2}, t_{i, 3} \cdots t_{i, i}$ which all keep ramifying the prime $q_{i+1,1}$ such that $\left(q_{i+1}\right)^{2^{i-1}}=\left(q_{i+1,1}\right)$ while all other primes remain inert. Note we can do this because there are only finitely many primes at each step. Fourth, we set $K_{i+1}=K_{i}\left[t_{i, 1}, t_{i, 2} \cdots t_{i, i}\right]$ and $D_{i+1}$ to be the integral closure of $D$ in $K_{i+1}$. Fifth, we set $D^{\infty}=\cup_{i=1}^{\infty} D_{i}$. Finally, $D^{\infty}$ is an almost Dedekind domain; moreover, it is a sequence domain. 
Thence, we know $D^{\infty}$ is a one-dimensional Prüfer domain. We need only show that no maximal ideal is idempotent to verify that $D^{\infty}$ is an almost Dedekind domain. Furthermore, $M^{*}=\left(q, q_{1,2}, q_{2,2} \cdots q_{i, 2}, \cdots\right)$ is the only maximal ideal that is not finitely generated. Additionally, $(q) \neq(q)^{2}$; thus, $q \notin\left(M^{*}\right)^{2}$. Hence, $M^{*}$ is not idempotent.

The rest of the maximal ideals are all principal; hence, they are not idempotent. Therefore, $D^{\infty}$ is an almost Dedekind domain. Further, $N(q)=\left(1 ; 1,2,4, \ldots, 2^{n}, \ldots\right)$, where the first entry is the value at $M^{*}$ and the other values are from $\left(q_{1}\right),\left(q_{2}\right), \ldots$, respectively.

Note a sequence domain is an almost Dedekind domain with a nonzero Jacobson radical such that there is exactly one non-finitely generated maximal ideal, and every other maximal ideal is principal and can be indexed by $\mathbb{N}$. See [6].

We start by proving a very useful lemma concerning prime elements in atomic domains.

Lemma 3.4. Let $D$ be an atomic domain and $\alpha \in D$ a prime element. Then $\cap_{n \in \mathbb{N}} \alpha^{n} D=\{0\}$ and $D\left[\alpha^{-1}\right]$ is atomic.

Proof. Assume that there exists some nonzero $x \in \cap_{n \in \mathbb{N}} \alpha^{n} D$. Now there exists some positive integer $l$ such that $x$ is a product of $l$ atoms. But, since $\alpha$ is prime, it is easy to see that $\alpha$ is associated with each of these atoms. Hence, $x$ is associated to $\alpha^{l}$. Consequently, $x \notin \alpha^{l+1} D$, a contradiction. Therefore, we conclude $\cap_{n \in \mathbb{N}} \alpha^{n} D=\{0\}$.

Now it is sufficient to show that every atom of $D$ which is not a unit of $D\left[\alpha^{-1}\right]$ is an atom of $D\left[\alpha^{-1}\right]$. Let $b$ be an atom of $D$ which is not a unit of $D\left[\alpha^{-1}\right]$. Let $c, d \in D\left[\alpha^{-1}\right]$ be such that $b=c d$. Since $\cap_{n \in \mathbb{N}} \alpha^{n} D=\{0\}$, there is some $m, n \in \mathbb{Z}$ and some $s, t \in D$ such that $\alpha$ is not a divisor of $s t, c=\alpha^{n} s, d=\alpha^{m} t$. It follows that $b=\alpha^{n+m} s t$. Since $\alpha$ is not a divisor of st and $b$ is not a unit of $D\left[\alpha^{-1}\right]$, we obtain that $b=s t$. Consequently, $s$ (or $t$ ) is a unit of $D$, and thus $c$ (or $d$ ) is a unit of $D\left[\alpha^{-1}\right]$.

We state one more lemma before moving onto the main theorem of this section. While the theorem we prove is interesting in its own regard, the corollaries that follow will also be of great interest. Throughout the rest of the paper $\mathcal{J}=\cap_{M \in \operatorname{Max}(D)} M$ will denote the Jacobson radical of $D$ and $\max (b)=\{M \in \operatorname{Max}(D) \mid b \in M\}$. 
Lemma 3.5. Let $D$ be an almost Dedekind domain with $\mathcal{J} \neq 0$, let $b$ be a nonzero element of $D$, and let $P, Q \in \max (b)$ be distinct. Then there exists a nonzero $c \in J$ such that, for each $m, n \in \mathbb{N}$, we have $\nu_{P}\left(c^{m} / b^{n}\right) \neq 0$ or $\nu_{Q}\left(c^{m} / b^{n}\right) \neq 0$.

Proof. Let $d \in \mathcal{J}$ be nonzero and $f \in P \backslash Q$. Set $c=d f^{\nu_{Q}(d) \nu_{P}(b)}$. Then $c \in \mathcal{J}$. Let $m, n \in \mathbb{N}$.

Case 1. $m \nu_{Q}(d) \geq n$. We have

$$
\begin{aligned}
\nu_{P}\left(c^{m} / b^{n}\right) & =m \nu_{P}(d)+m \nu_{Q}(d) \nu_{P}(b) \nu_{P}(f)-n \nu_{P}(b) \\
& >n \nu_{P}(b)\left(\nu_{P}(f)-1\right) \geq 0 .
\end{aligned}
$$

Case 2. $m \nu_{Q}(d)<n$. We have

$$
\begin{aligned}
\nu_{Q}\left(c^{m} / b^{n}\right) & =m \nu_{Q}(d)+m \nu_{Q}(d) \nu_{P}(b) \nu_{Q}(f)-n \nu_{Q}(b) \\
& <n\left(1-\nu_{Q}(b)\right) \leq 0 .
\end{aligned}
$$

We are now in a position to prove a theorem about bounded atoms. The reader should note that this is a generalization of the primes of a Dedekind domain with nonzero Jacobson radical.

Theorem 3.6. Let $D$ be an almost Dedekind domain with $\mathcal{J} \neq 0$. Then every bounded atom of $D$ is a prime element.

Proof. Let $b$ be a bounded atom of $D$. Then there exists some $\rho \in \mathbb{N}$ such that $\nu_{M}(b)<\rho$ for all $M \in \operatorname{Max}(D)$. First we show that $\max (b)$ is a singleton set. Assume, to the contrary, that there are distinct $P, Q \in \max (b)$. By the previous lemma, there is some nonzero $c \in \mathcal{J}$ such that, for all $m, n \in \mathbb{N}$, it follows that $\nu_{P}\left(c^{m} / b^{n}\right) \neq 0$ or $\nu_{Q}\left(c^{m} / b^{n}\right) \neq 0$. For $M \in \operatorname{Max}(D)$, set

$$
d_{M}=\min \left\{\nu_{U}(c) \mid U \in \operatorname{Max}(D), \nu_{U}(b)=\nu_{M}(b)\right\} .
$$

Clearly, $\left\{d_{M} \mid M \in \operatorname{Max}(D)\right\}$ is finite. Set

$$
d=\max \left\{\frac{\nu_{M}(b)}{d_{M}} \mid M \in \operatorname{Max}(D)\right\} .
$$


We have $d=\nu_{U}(b) / d_{U}$ for some $U \in \max (b)$. There is some $L \in \max (b)$ such that

$$
\nu_{L}(b)=\nu_{U}(b)
$$

and

$$
d_{U}=\nu_{L}(c)
$$

Set $m=\nu_{L}(b)$ and $n=\nu_{L}(c)$. Let $M \in \operatorname{Max}(D)$. Then $d_{M} \leq \nu_{M}(c)$, and thus

$$
\frac{\nu_{M}(b)}{\nu_{M}(c)} \leq \frac{\nu_{M}(b)}{d_{M}} \leq d=\frac{\nu_{U}(b)}{d_{U}}=\frac{m}{n} .
$$

This implies that $\nu_{M}\left(b^{n}\right)=n \nu_{M}(b) \leq m \nu_{M}(c)=\nu_{M}\left(c^{m}\right)$; hence, $N\left(b^{n}\right) \leq N\left(c^{m}\right)$.

Set $f=c^{m} / b^{n}$. Then $f \in D$. Moreover, $\nu_{P}(f) \neq 0$ or $\nu_{Q}(f) \neq 0$.

If $M \in \max (f)$, then

$$
\nu_{M}(b)<\rho \leq \rho \nu_{M}(f)=\nu_{M}\left(f^{\rho}\right) .
$$

If $M \notin \max (f)$, then $n \nu_{M}(b)=m \nu_{M}(c)>0$; hence,

$$
\nu_{M}\left(f^{\rho}\right)=\rho \nu_{M}(f)=0<\nu_{M}(b) .
$$

We infer

$$
\nu_{M}\left(b+f^{\rho}\right)= \begin{cases}\nu_{M}(b) & M \in \max (f) \\ 0 & M \notin \max (f) .\end{cases}
$$

Since $\nu_{L}(f)=0$, we have $\nu_{L}\left(b+f^{\rho}\right)=0<\nu_{L}(b)$, and thus

$$
N\left(b+f^{\rho}\right)<N(b) .
$$

Consequently, $b+f^{\rho}$ is a unit of $D$ (since b is an atom of $\left.D\right)$. Therefore, $P \notin \max (f)$ and $Q \notin \max (f)$, a contradiction. It follows that $\max (b)=\{M\}$ for some $M \in \operatorname{Max}(D)$. We obtain that $M$ is a sharp maximal ideal of $D$, and thus $M=(c)$ for some $c \in M$ (See [7, Corollary 2.7].) Therefore, $(b) \subseteq(c) \subset D$; hence, $M=(b)$. We conclude that $b$ is a prime element of $D$.

We immediately get some nice corollaries. 
Corollary 3.7. Let $D$ be an atomic bounded almost Dedekind (not Dedekind) domain. Then $\mathcal{J}=\{0\}$.

Proof. Assume $\mathcal{J} \neq\{0\}$. Then, by the previous theorem, we obtain that $D$ is a UFD; hence, $D$ is a PID, a contradiction.

Corollary 3.8. A glad domain is atomic if and only if it is a semi-local PID.

Proof. A glad domain is bounded and has a nonzero Jacobson radical. Hence, if it is atomic it must be Dedekind. A Dedekind domain with finitely many primes is a semi-local PID.

Corollary 3.9. If $D$ is an atomic SP-domain (not a Dedekind domain), then $\mathcal{J}=0$.

We see from the above theorem that $\mathcal{J}$ plays an important role in determining atomicity. This motivates the study of almost Dedekind domains with nonzero Jacobson radical.

Now we see that, if $D$ is an atomic almost Dedekind domain with $\mathcal{J} \neq 0$, then $D$ must not have too many sharp primes, for the generator of every sharp prime is an atom. If we have too many atoms of this form, it will be hard to keep an atomic factorization finite. This is stated more precisely in the next lemma.

Lemma 3.10. If $D$ is an atomic almost Dedekind domain with $\mathcal{J} \neq 0$, then $D$ can only have finitely many sharp primes.

Proof. Suppose $D$ has infinitely many sharp primes. Let $0 \neq d \in \mathcal{J}$. Since $D$ is atomic we factor $d$ into atoms as $d=\alpha_{1} \alpha_{2} \cdots \alpha_{n}$. Now $\alpha_{i}$ must be in infinitely many sharp primes for some $i$. But the generator $m_{i}$ of $M_{i}$ divides $\alpha_{i}$ for all sharp primes $M_{i}$. Thus, this factorization is not an atomic factorization. We conclude that $D$ has only finitely many sharp primes.

Corollary 3.11. There does not exist an atomic sequence domain. 
Now that we know that an atomic almost Dedekind domain with $\mathcal{J} \neq 0$ has only finitely many sharp maximal ideals, one might ask what role these sharp maximal ideals play. ince these sharp maximal ideals are principal ([7, Corollary 2.7]) and generated by a prime element, we see from Lemma 3.4 that we can remove them by turning the prime elements into units, further we can do this in a way that the resulting domain is still atomic.

An almost Dedekind domain that contains only dull maximal ideals will be called a dull domain. We will see that we can reduce our study of atomicity in almost Dedekind domains with nonzero Jacobson radicals to the study of dull domains with nonzero Jacobson radicals. It should be noted that overrings of almost Dedekind domains with nonzero Jacobson radicals are almost Dedekind domains with nonzero Jacobson radicals; further, it should be noted that we are excluding domains from being fields.

Theorem 3.12. If $D$ is an atomic almost Dedekind (not Dedekind) domain with $\mathcal{J} \neq 0$, then there exists a dull domain $D^{\prime}$ derived from $D$ that remains atomic.

Proof. If $D$ has no sharp primes there is nothing to prove. If $D$ has sharp primes they are all principal. Moreover, there can only be finitely many principal primes. Let $\left(\alpha_{1}\right),\left(\alpha_{2}\right), \ldots\left(\alpha_{n}\right)$ be the list of sharp primes. Now we apply the previous Lemma 3.4 a finite number of times to arrive at

$$
D^{\prime}=D\left[\alpha_{1}^{-1}, \alpha_{2}^{-1}, \cdots \alpha_{n}^{-1}\right]
$$

Now we know $D^{\prime}$ is an atomic domain. Furthermore, $D^{\prime}$ has no sharp primes, for we annihilated the sharp primes.

Recall an antimatter domain is a domain that contains no atoms. For more on antimatter domains see [2].

We introduce a new type of domain that seems highly unusual.

Definition 3.13. We call an almost Dedekind domain $D$ (not a field) completely unbounded, if for all nonzero non-units $b \in D$ we have $b$ unbounded. We will call these completely unbounded domains. 
Corollary 3.14. Let $D$ be a dull domain with $\mathcal{J} \neq\{0\}$.

(i) If $D$ is bounded, then $D$ is an antimatter domain.

(ii) If $D$ is atomic, then $D$ is completely unbounded.

Proof. Since $D$ is a dull domain, it follows that $D$ does not contain any prime elements. Therefore, $D$ does not contain any bounded atoms by Theorem 3.6.

(i) Let $D$ be bounded. It is immediately clear that $D$ is an antimatter domain.

(ii) Let $D$ be atomic and $b$ a nonzero non-unit of $D$. Clearly, $b$ is divisible by some atom $\alpha$ of $D$. Since $\alpha$ is unbounded, we infer that $b$ is unbounded.

Combining Theorem 3.12 and Corollary 3.14 we achieve the following theorem.

Theorem 3.15. If there exists an atomic almost Dedekind (not Dedekind) domain with $\mathcal{J} \neq\{0\}$, then there exists an atomic completely unbounded almost Dedekind domain.

It is not clear at the present whether one can construct a completely unbounded almost Dedekind domain. Even if one can construct a completely unbounded domain, can one construct an atomic completely unbounded domain? If no such completely unbounded domains exist, then we would be able to conclude that an almost Dedekind domain with a nonzero Jacobson radical is atomic if and only if it is Dedekind.

Acknowledgments. The author would like to thank the referees for their invaluable input on this work.

\section{REFERENCES}

1. J. Coykendall, Normsets and determination of unique factorization in rings of algebraic integers, Proc. Amer. Math. Soc. 124 (1996), 1727-1732.

2. J. Coykendall, D. Dobbs and B. Mullins, On integral domains with no atoms, Comm. Alg. 27 (1999), 5813-5831.

3. R. Gilmer, Multiplicative Ideal Theory, Queen's Papers Pure Appl. Math. 90, Queen's University Press, Kingston, 1992. 
4. A. Grams, Atomic rings and the ascending chain condition for principal ideals, Proc. Camb. Phil. Soc. 75 (1974), 321-329.

5. K.A. Loper, Almost Dedekind domains which are not Dedekind, in Multiplicative ideal theory in commutative algebra; A tribute to the work of Robert Gilmer, James W. Brewer, Sarah Glaz, William J. Heinzer and Bruce M. Olberding, eds., Springer, New York, 2006.

6. __ Sequence domains and integer-valued polynomials, J. Pure Appl. Alg. 119 (1997), 185-210.

7. K.A. Loper and T.G. Lucas, Factoring ideals in almost Dedekind domains, J. reine angew. Math. 565 (2003), 61-78.

8. B. Olberding, Factorization into radical ideals, in Arithmetical properties of commutative rings and monoids, Lect. Notes Pure Appl. Math. 241, Chapman \& Hall/CRC, Boca Raton, FL, 2005.

600 N Grand Ave, Tahlequah, OK 74464

Email address: hasenaue@nsuok.edu 